state that Glegg's book uses "design" in the sense of the second area. This is implicitly the only place where design takes place. It is a rather boring place where "there is no unsophisticated engineering left, or there shouldn't be". What this means is that most of the basic devices or ways of doing things have already been invented or discovered. From this notion derives the term "selection" in the title of the book. The activity of design is largely concerned with the selection of possible ways of doing things from an apparently existing catalogue. From the many possibilities one eliminates by selection by context and by selection of content. Somehow economics takes a back seat. Very occasionally there are apparent impossibilities to tackle. This is the creative activity.

Within his limits Glegg has produced a very interesting little book which is essentially of the "how I" rather than the "how to" kind. Whether it will be suitable to young inventors will depend upon their lines of activity. In some ways they may have to be interested in the bits and pieces of motor cars. But there is no suggestion of further reading or hints of what other people have been doing in the design business and how his ideas compare with theirs.

\section{SYDNEY GREGORY}

\section{Photosynthetic Enzymes}

Methods in Enzymology, Photosynthesis and Nitrogen Fixation. Edited by Anthony San Pietro. Volume 24, Part B. Pp. vii +526. (Academic: London and New York, September 1972.) $\$ 23.50$.

AN up-to-date method book for photosynthesis research has been needed for a long time. The present book fills this need by giving an almost full account of a vast number of experimental approaches, ranging from biochemical to physical, to this multi-faceted subject.

Of the two volumes dealing with photosynthesis and nitrogen fixation, part A has been devoted to preparatory methods. Part B, which is the matter of this review, is less homogenous; its main body is devoted to processes and measurements, but it includes preparative aspects as well in the sections on "Synthesizing Capabilities" and "Nitrogen Fixation".

The book is divided into four sections, containing forty-four chapters, each dealing with a different methodological aspect and written by a different author. Although this guarantees, in general, authority and a high standard of presentation, the book as a whole suffers from a lack of homogeneity and coherence. This could be perhaps eliminated by elaborate editing and planning. The various chapters should have been grouped together according to being physical, chemical or biochemical, and further subgrouped into various sub-categories. For example, optical and photophysical methods could be grouped together, with an appropriate unifying introduction. The same is true with other categories. The division to small chapters also causes unnecessary repetition. For example, light intensity measurements are included in the chapters "Quantum Yields" and "Light Intensity Measurements". There are other examples of this.

The level and depth of the presentation are not equal. Besides very comprehensive treatises (to mention but a few: "Flash Kinetic Spectrophotometry", "Light Induced Paramagnetism", "Measurement of Hill Reaction") there are some small chapters that miss essential details. For example, methods of treating data in order to obtain the limiting quantum yield are absent; the chapter "Enhancement", besides defining the subject, gives just little more than how to measure a Hill reaction, and particular aspects regarding how to treat the data and methods to deal with non-linearity of rate with intensity (for example, the Kok effect) are not expanded; the small note on "A Green Safelight" could be expanded and included in the chapter on "Light Sources and Measurements"; the coverage of fluorescence and delayed light methods is too brief; the details of measuring $\mathrm{O}_{2}$ evolution from single flashes, and the detection of other redox reaction by the oxygen electrode, although mentioned, are also too brief.

There are descriptions of very specific methods, which have not been extensively and independently reviewed. For example, the method of high derivative absorption spectrum to separate peaks in a complicated spectrum should be applied with caution (since each derivative adds, in principle, an additional peak to the original spectrum) and probably is valid only under limited conditions. Also, the method of steadystate relaxation spectrophotometry, although a very general tool in chemical kinetics, seems to have only a limited application here.

The coverage of the typical biochemical and preparative chapters seems adequate. In fact, the writing of a typical biochemical method involves far fewer problems and is more or less standardized compared to the writing of a physical method.

These drawbacks, serious as they are, do not impair the usefulness of the book, which serves as a quick reference and source for most of the methods existing in the field, and is thus very welcome.

\section{Extinction by Hormone}

Insect Juvenile Hormones: Chemistry and Action. Edited by Julius J. Menn and Morton Beroza. (Proceedings of a Symposium held in Washington, September 1971.) Pp. $\quad x v+341$. (Academic: New York and London, June 1972.) n.p.

Insect Sex Pheromones. By Martin Jacobson. Pp. xii +382. (Academic: New York and London, October 1972.) $\$ 22.50$.

JUVENILE hormone functions during insect metamorphosis by actively favouring the differentiation of larval characters, and in many species it also controls oocyte vitellogenesis in the adult female. If the corpora allata-the glands which produce juvenile hormone -are removed from early larval instars, dwarf adults can result, and if the hormone is introduced when it is normally absent, supernumerary larval instars or monsters intermediate between larva, pupa and adult can be produced. Moreover, when juvenile hormone is applied to the eggs of some insects, embryogenesis can be abnormal or postembryonic development can be affected. It is not surprising, therefore, that the prediction was made that juvenile hormone could prove to be a potent insecticide-with the advantages over current synthetic insecticides that, being a natural compound, it would be unlikely to have adverse environmental effects, and that the insects would be unlikely to develop immunity to one of their own hormones.

Juvenile hormone was discovered nearly forty years ago, and its chemical identity was established in 1967 as methyl 10,11-epoxy-7-ethyl-3,11-dimethyl-2-6-tridecadienoate. A second hormone, with a methyl group replacing the ethyl at $C_{7}$, was described a short time later. In the years immediately before the identification of juvenile hormone, other chemicals were discovered which mimicked its activities, and families of compounds are now known which have greater or less juvenile hormone activity in a variety of insect species. These discoveries raised the possibility that hormonomimetic chemicals could be tailored for use against specific insect pests.

Insect Juvenile Hormones discusses these aspects of juvenile hormone and its mimics in the light of recent evidence. The volume contains contributions on the chemistry, biochemistry, metabolism and action of the authentic hormones, and of many hormonomimetic chemicals. Preliminary field trials of some of the compounds are described, together with tests on their environmental stability and decomposition products. Very brief details of their toxicity to mammals and plants are also provided. 